\title{
Diversity-orientierte Führung - Erfolgsfaktor im Mint-Bereich?
}

\author{
Petia Genkova $\cdot$ Henrik Schreiber
}

Eingegangen: 2. Juli 2021 / Angenommen: 3. August 2021 / Online publiziert: 29. Oktober 2021

(C) Der/die Autor(en) 2021

Zusammenfassung Diese qualitative Studie untersucht explorativ MINT-Führungskräfte hinsichtlich ihrer Erfahrungen, sozialer Einflüsse und Kompetenzen, die für den Umgang mit kultureller Vielfalt relevant sind. Die Ergebnisse zeigen, dass sich nur ein kleiner Teil der Befragten der Chancen und Risiken kultureller Vielfalt und der spezifischen Herausforderungen für Menschen mit Migrationshintergrund bewusst ist. Dabei scheinen für die Ausbildung der Einstellungen sowohl die eigenen Erfahrungen und die Sensibilität, die eigene Identifizierung mit dem Unternehmen vor dem Hintergrund der jeweiligen Position des Unternehmens und bisherige Maßnahmen relevant zu sein und miteinander zu interagieren.

Schlüsselwörter Diversity-Management · MINT · Diversity-Einstellungen

\section{Diversity-oriented leadership-Success factor in the STEM sector?}

\begin{abstract}
The present qualitative study examines the experiences, social influences, and competencies of managers in the STEM sector, which are important for managing cultural diversity. The results demonstrate that few of the interviewees are aware of the chances and risks of cultural diversity and the specific challenges for people with a migration background. Furthermore, one's own experiences, and sensitivity, one's own identification with the company and previous diversity measures in the context of the companies' position seem to be relevant for the formation of attitudes.
\end{abstract}

Keywords Diversity management $\cdot \mathrm{STEM} \cdot$ Diversity attitude

Prof. Dr. Petia Genkova $(\bowtie) \cdot$ Henrik Schreiber

Hochschule Osnabrück, Caprivistr. 30 A, 49076 Osnabrück, Deutschland

E-Mail: petia@genkova.de

Henrik Schreiber

E-Mail: henrik.schreiber@hs-osnabrueck.de 


\section{Diversity-Einstellung von MINT-Führungskräften}

Strukturen, die sich durch eine hohe Heterogenität ihrer Mitglieder auszeichnen, sind durchschnittlich erfolgreicher, wenn es gelingt, die vorhandene Vielfalt an Kompetenzen und Sichtweisen zu nutzen (van Dick und Stegmann 2016). Dies ist das Ziel der Ansätze und Methoden, die unter dem Begriff Diversity-Management zusammengefasst werden. Abhängig davon, wie mit Vielfalt in Arbeitsgruppen umgegangen wird, führt kulturelle Diversität zu besseren Individual- und Teamleistungen (Stegmann 2011). Laut dem Institut der deutschen Wirtschaft zeichnet sich insbesondere die MINT-Branche (Mathematik, Informatik, Naturwissenschaften, Technik) durch eine hohe internationale Mobilität auf dem Arbeitsmarkt und einen hohen Anteil (19\%) ausländischer Expert/innen in deutschen Unternehmen aus (Anger et al. 2018), zuzüglich der Personen, deren Eltern nach Deutschland eingewandert sind. Diversity-Management ist in der MINT-Branche folglich besonders wichtig, um Leistungs- und Wettbewerbsfähigkeit sicherzustellen. Genkova und Schreiber (2019) betonen in ihrem Konferenzbeitrag zur Wahrnehmung von Aspekten kultureller Vielfalt, dass Führungskräfte dabei eine entscheidende Rolle spielen. Während das Top-Management eine generelle Strategie und öffentliche Position entwickelt, sind gehobene und mittlere Führungskräfte gefordert, diese Strategie vorzuleben, indem sie z. B. Offenheit und Flexibilität demonstrieren. Sie werden daher auch als Multiplikator/innen des Diversity-Managements beschrieben (Wildermuth und Gray 2005).

Gleichzeitig fehlen Erkenntnisse darüber, welche Einstellungen zu kultureller Vielfalt bei Führungskräften vorhanden sind und wie sie sich entwickeln oder verändern. Dies gilt insbesondere für technische und naturwissenschaftliche Berufsgruppen. Ein effizienter Umgang mit Vielfalt ist hier weder in Curricula (Koller und Rudolph 2017) noch im öffentlichen Bewusstsein etabliert (Anger et al. 2018), und Forschungsergebnisse fehlen. Die vorliegende Studie hat aus diesem Grund eine explorative Betrachtung der Einstellungen, Erfahrungen, Kompetenzen und des sozialen Umfeldes von MINT-Führungskräften vorgenommen.

\subsection{Kulturelle Vielfalt, Identität und Leistung}

Thomas (2003) beschreibt Kultur als psychologisches Orientierungssystem, das Zugehörigkeit durch die Wahrnehmung, das Denken und Handeln der Mitglieder definiert. Kultur ist für ein Individuum ein Aspekt der sozialen Identität und wird herangezogen, um sich selbst und andere in Kategorien einzuteilen, was mit einer stärkeren Akzentuierung subjektiv relevanter Unterschiede zwischen Gruppen einhergeht (Social-Identity-Ansatz; vgl. Tajfel und Turner 1979). In dem Moment, in dem eine dieser Gruppen salienter, bzw. subjektiv relevanter, wird, neigen Menschen dazu, sich selbst und andere weniger als Individuen, sondern als Repräsentant/innen einer einzelnen Gruppe zu betrachten (van Dick und Stegmann 2016).

Das Categorization-Elaboration-Modell (CEM) (van Knippenberg et al. 2004) basiert auf dem Verständnis von Identität in Anlehnung an den Social-Identity-Ansatz und war das erste Modell, das die Leistungsunterschiede zwischen verschiedenen diversen Teams replizierbar erklären konnte (van Dick und Stegmann 2016). Die Kern- 
Annahme des CEM-Modells ist, dass Gruppen mit diversen Mitgliedern ein höheres Potenzial an Sichtweisen, Kenntnissen oder allgemein Informationen haben, wenn mit entsprechenden Subgruppen effizient zusammengearbeitet wird. Die Informationsvielfalt soll idealerweise zu einer vertieften Informations-Elaboration und damit zu besser durchdachten oder innovativeren Lösungen führen. Eine Subgruppenbildung (subjektiv relevante Gruppierungsmerkmale und Salienz dieser Gruppierung) führt dann zu negativen affektiven Konsequenzen und folglich zu schlechterer Performance, wenn die Subgruppen zueinander ein feindseliges Verhältnis haben. Dies entsteht nach diesem Modell dann, wenn sich die Subgruppen gegenseitig oder ihren jeweiligen Beitrag nicht anerkennen, sich also in ihrer Identität bedrohen (van Dick und Stegmann 2016).

\subsection{Diversity-Management und Führung}

Van Dick und Stegmann (2016) legen in ihrem Übersichtskapitel dar, dass die Herausforderung für moderne, diverse Teams und Gesellschaften daher nicht die Diversität ihrer Mitglieder an sich ist und auch nicht die unvermeidliche subjektive Relevanz von Unterschieden - Faultlines (Bruchkanten) - zwischen Subgruppen. Die wichtigste Aufgabe des Diversity-Managements besteht vielmehr darin, eine etwaige bedrohliche Beziehung zwischen den Gruppen durch eine produktive und sinnvolle Beziehung zu ersetzen (van Dick und Stegmann 2016). Dies erfordert eine positive geteilte Einstellung zu Vielfalt, aber auch Fähigkeiten zu einer kooperativen, wertschätzenden Zusammenarbeit und Führung.

Grundlegend für Zusammenarbeit ist eine übergeordneten Identität, die Kohäsion und Commitment steigert (van Knippenberg und Schie 2000). Dies erfordert auf Grundlage der Eigengruppenprojektionshypothese (Mummendey und Wenzel 1999) auch ein Bewusstsein dafür, dass die übergeordnete Gruppe, das Team oder die Organisation nicht kulturell homogen ist, sondern durch die Vielzahl unterschiedlicher Perspektiven zu dem wird, was sie ist. Gutentag et al. (2018) zeigen darauf aufbauend, dass die Grundlage für diversitätssensibles Verhalten ein differenziertes Bewusstsein für kulturelle Vielfalt ist. Dieses verläuft auf einem Spektrum zwischen den Perspektiven culture-blind (Annahme, dass es keine kulturellen Unterschiede gibt, alle Menschen sind gleich) und color-ful (Berücksichtigung kultureller Unterschiede; Cox 1991), was sowohl von Organisationen als auch von Einzelpersonen vertreten werden kann. Darüber hinaus sind die individuellen Einstellungen gegenüber Vielfalt entscheidend. Der Begriff Diversitätsüberzeugungen (engl. diversity beliefs) wurde ursprünglich von van Knippenberg und Haslam (2003) eingeführt zur Bezeichnung der individuellen Überzeugungen, dass Vielfalt für eine Gruppe vorteilhaft ist.

Die aktuelle Literatur im Bereich Diversity-Management stellt umfassend dar, wie Einstellungen zu kultureller Vielfalt bei Angestellten durch die Verhaltensweisen von Führungskräften beeinflusst werden (für einen Überblick vgl. Genkova 2019). Ashikali und Groeneveld (2015) zeigen anhand einer sehr großen Studie mit über zehntausend Teilnehmenden, dass die Wahrnehmung von kultur-fairen Auswahlverfahren durch die Angestellten, die Wahrnehmung von Wertschätzung und einem bewussten, kompetenten Umgang von Führungskräften mit kultureller Viel- 
falt signifikant positiv mit den Diversity-Einstellungen sowie mit dem Commitment der jeweiligen Angestellten zusammenhängen. Dieser Zusammenhang wird teilweise mediiert durch einen transformationalen Führungsstil (Ashikali und Groeneveld 2015). Sie erklären diese Beziehung dadurch, dass Angestellte generell, und bei einem transformationalen Führungsstil insbesondere, Einstellungen und Verhaltensweisen im Sinne der Ausbildung einer Diversity-Kultur übernehmen. Die DiversityKultur, d.h. ein sozial geteiltes Set an Meinungen und Verhaltensweisen (Schein 2010) hinsichtlich der Einschätzung des Umgangs mit (in diesem Fall kultureller) Vielfalt, ist dabei ein zentraler Prädiktor der Leistung heterogener Arbeitsgruppen (Kundu und Mor 2017). Dies zeigte auch Stegmann (2011) in einer umfassenden Metaanalyse. Führungskräfte stehen dabei generell im Spannungsfeld, unter Berücksichtigung ihrer übrigen Führungsaufgaben und möglichen fachlichen Aufgaben die Zusammenhänge und Relevanz von Diversity-Management zu verstehen, Sensibilität zu entwickeln und möglicherweise eigene Animositäten und Unsicherheiten hinter sich zu lassen (Ashikali und Groeneveld 2015; Genkova 2019; McCallaghan et al. 2020).

Während Verhaltensweisen von Führungskräften durch die Befragung von Angestellten verhältnismäßig einfach untersucht werden können, gibt es bisher nur sehr wenige Studien, die sich den Einstellungen und Perspektiven von Führungskräften widmen, obwohl diese, wie oben dargestellt, als Multiplikator/innen von erfolgreichem Diversity-Management verstanden werden können. Wie in der Einleitung beschrieben, sind diese Erkenntnisse besonders für die MINT-Branche entscheidend, die mehr als jeder andere Bereich durch kulturelle Vielfalt geprägt ist (Anger et al. 2018). Obwohl davon auszugehen ist, dass Kompetenzen, Einstellungen und Perspektiven von MINT-Absolvent/innen von denen von Personen mit geistesoder wirtschaftswissenschaftlicher Ausbildung abweichen (Canagarajah 2018), liegen gerade für Führungskräfte der MINT-Branche keine differenzierten Ergebnisse vor. Ziel dieser Studie ist es daher, zum besseren Verständnis von Einstellungen zu kultureller Vielfalt von Führungskräften in MINT-Berufen beizutragen. Dafür soll einerseits exploriert werden, welche Einstellungen zu kultureller Vielfalt bei den befragten Führungskräften vorhanden sind. Zudem werden soziale Einflüsse, die umgebende Diversity-Kultur sowie Kompetenzen, die für den Umgang mit Vielfalt hilfreich sein können, betrachtet. Es wurden die folgenden Leitfragen formuliert:

1. Welche Einstellungen zu kultureller Vielfalt gibt es bei den untersuchten MINTFührungskräften?

2. Welche Erfahrungen sind bei MINT-Führungskräften für die Einstellungen zu kultureller Vielfalt besonders relevant?

3. Welche Einstellungen und Perspektiven im sozialen Umfeld sind bei MINT-Führungskräften für die Einstellungen zu kultureller Vielfalt besonders relevant?

4. Welche Kompetenzen und welches Knowhow sind bei MINT-Führungskräften vorhanden und für die Einstellungen zu kultureller Vielfalt besonders relevant? 


\section{Methode}

Da für diesen spezifischen Bereich kaum Erkenntnisse vorliegen, wurde ein qualitatives, exploratives Vorgehen genutzt. Die strukturierten Interviews sollten ein Bild der subjektiven Theorien (Hilmer 1969) der Führungskräfte aus dem MINT-Bereich zu Diversity-Einstellungen und interkultureller Kompetenz ermöglichen. Der Interview-Leitfaden beinhaltete insgesamt 58 Fragen. Um konzeptuelle Äquivalenz und Vergleichbarkeit (Genkova 2019) über die Subgruppen (Führungskräfte mit und ohne Migrationshintergrund, mit viel und wenig Erfahrung) hinweg zu gewährleisten, wurde der fertige Interview-Leitfragen von mehreren Experten für Diversity mit und ohne Migrationshintergrund diskutiert und nach kleineren Änderungen hinsichtlich allgemein gefasster Formulierungen freigegeben.

\subsection{Untersuchungsdurchführung}

Die Interviews fanden im Zeitraum Januar bis März 2020 statt. Über wissenschaftliche und wirtschaftliche Netzwerke wurden Führungskräfte aus verschiedenen Unternehmen, die einen Abschluss in MINT-Fächern haben und in diesem Bereich arbeiten, für die telefonischen Interviews gewonnen. In Übereinstimmung mit den Datenschutzrichtlinien wurde das ausdrückliche Einverständnis eingeholt, dass die Interviews aufgezeichnet und zu ausschließlich wissenschaftlichen Zwecken verwendet werden dürfen. Die Interviews dauerten zwischen 20 und 45 min. Eine Aufwandsentschädigung erfolgte nicht. Die aufgezeichneten Interviews wurden transkribiert und mittels qualitativer Inhaltsanalyse nach Mayring (2015) induktiv ausgewertet. Die Kategorienbildung wurde mit drei Diversity-Expert/innen an Hochschulen diskutiert. Dies entspricht einem von Bengtsson (2016) vorgeschlagenen Vorgehen zur Triangulation.

\subsection{Teilnehmende}

Es wurden insgesamt 22 Führungskräfte verschiedener deutscher Unternehmen befragt. Die Befragten waren zwischen 26 und 69 Jahren alt $(M=49)$. Drei der Befragten waren weiblich, 19 männlich. Alle Befragten hatten einen Hochschulabschluss in einem MINT-Fach. Drei der Befragten hatten einen Migrationshintergrund (nach der Definition von Kemper (2010) Menschen, die selbst oder bei denen mindestens ein Elternteil nach Deutschland eingewandert sind). Zehn Befragte ordneten sich selbst auf der mittleren Führungsebene ein, zwölf auf der oberen Führungsebene. Fünf Befragte $(23 \%)$ gaben an, in einem kleinen Unternehmen zu arbeiten (bis zu 50 Angestellte), acht (36\%) gaben an, in einem mittelgroßen Unternehmen (bis zu 250 Angestellte) und neun (41\%), in einem großen Unternehmen mit mehr als 250 Angestellten zu arbeiten. Davon waren acht Unternehmen in dem Bereich IT und Software tätig, sechs im Hoch-, Tief-, oder Anlagenbau, zwei in der Stahl- und Baustoff-Produktion, zwei im Consulting und je eines in den Bereichen Automobil, Energiewirtschaft, Holzverarbeitung und Nahrungsmittel. Die Führungskräfte schätzten den Anteil an Personen mit Migrationshintergrund im Schnitt mit 17,5\% ein (vgl. Tab. 1). 
Tab. 1 Geschätzter Anteil an Personen mit Migrationshintergrund in den Unternehmen nach Größe

\begin{tabular}{llllll}
\hline & & \multicolumn{2}{l}{ Anteil der Personen mit Migrationshintergrund } & Gesamt \\
& & $<10 \%$ & $<30 \%$ & $>30 \%$ & \\
\hline Unternehmensgröße & Klein & 2 & 2 & 1 & 5 \\
& Mittel & 3 & 3 & 2 & 8 \\
& Groß & 2 & 5 & 2 & 22 \\
\hline
\end{tabular}

\section{Ergebnisse}

\subsection{Welche Einstellungen zu kultureller Vielfalt gibt es bei den untersuchten MINT-Führungskräften?}

Um die Frage zu beantworten, welche Einstellungen zu kultureller Vielfalt bei den untersuchten Führungskräften generell vorhanden sind, wurden verschiedene aufeinander aufbauende Fragen gestellt, die wahrgenommene und vermutete Vorteile und Nachteile kultureller Vielfalt in Unternehmen aufdecken sollten. Es zeigt sich, dass fünf Befragte mehrere Antworten gaben, in denen sie verschiedene Aspekte von Vielfalt und auch indirekte Vor- und Nachteile diskutierten (Kategorien: Arbeitsmotivation, Offenheit, Effizienteres Arbeiten, Fördert die Integration, Gegenseitiges Lernen, Zusätzliche Kompetenzen). Beispielsweise konnte sich ein Teilnehmer zwar besser in Personen ohne Migrationshintergrund hineinversetzen und war sich möglicher kultureller Missverständnisse bewusst, betonte aber die Rolle von Vielfalt für das Unternehmensklima und die Leistung der Angestellten. ,(...) da sind eben jeden Tag andere Menschen, die unterschiedliche Hintergründe haben, teilweise kulturelle Hintergründe, aber einfach auch aus unterschiedlichen Lebenssituationen kommen. Und wenn wir da offen sind, ähm, also wenn wir das intern schon lernen (...), dann können wir diese positiven Erfahrungen auch für Offenheit gegenüber Externen, also gegenüber unseren Kunden nutzen und daraus lernen." Demgegenüber konnten neun der 16 übrigen Befragten gar keine oder nur eine knappe Antwort geben, die sich auf direkte Konsequenzen von Diversität bezog (Kategorien: Zugang zu anderen Märkten (international und zu Migrierten), Sprachen, andere Perspektiven, Arbeitsweisen). Unsicherheit schien sich in kurzen, abgehackten Antworten zu zeigen, die eher allgemeine Positionen aufgriffen, teilweise kombiniert mit der Aufforderung, das Interview fortzusetzen (z. B. „Ähm, Verschiedenartigkeit bringt Vorteile (...). Gerade bei Projektarbeiten. Machen Sie weiter!").

Auffällig ist, dass 17 der befragten Führungskräfte an verschiedenen Stellen im Interview die Meinung äußerten, dass ,alle gleich“ seien und - in sechs Fällen - man daher besonderen Wert darauf lege, alle gleich zu behandeln, weshalb man auch keine besonderen Maßnahmen ergreife, um kulturelle Vielfalt und Gleichberechtigung zu unterstützen. Im Kontrast dazu geben zwölf der Führungskräfte an, sie hätten den Eindruck, dass sich Personen mit Migrationshintergrund am besten mit anderen Personen mit Migrationshintergrund verstehen. Bezüglich der Wahrnehmung zeigt sich darüber hinaus, dass viele der befragten Führungskräfte Personen mit Migrationshintergrund nicht als Teil der gleichen Hierarchiestufe sehen. Sie betonen z.B., 
dass Probleme interkultureller Interaktion in ihrem Unternehmen primär bei LagerAngestellten und Lastkraftfahrer/innen vorkommen. Diese 12 Führungskräfte sind in allen Fällen ebenfalls der Meinung, dass die wichtigste Fähigkeit für Personen mit Migrationshintergrund in ihrem Unternehmen Deutschkenntnisse sind.

Sieben der Führungskräfte sind der Meinung, dass Personen mit Migrationshintergrund leistungsbereiter oder disziplinierter sind als Personen ohne Migrationshintergrund. Dahinter scheint in den meisten Fällen die Einstellung zu stehen, dass Personen, die weniger oder kürzer die Annehmlichkeiten der westlichen Gesellschaft genießen durften, verbissener arbeiten. Illustriert wird das durch die sehr prägnante Formulierung: ,Ja, es gibt Unterschiede. Und das hängt sicherlich damit zusammen, wie man aufgewachsen ist und unter welchen Umständen man aufgewachsen ist. Wenn denn/jemand, dem schon einmal Kanonenkugeln um die Ohren geflogen sind, der ist sicherlich anders unterwegs als jemand, der wohlbehütet hier im Westen der Republik aufgewachsen ist."

Sechs Führungskräfte zeigen ein differenziertes Bild von den Vorteilen und Risiken kultureller Vielfalt in ihren Unternehmen. Sie sind der Meinung, dass sich die Herausforderungen und Stresszustände von Angestellten mit Migrationshintergrund unterscheiden, und beschreiben sowohl Chancen als auch Risiken für Personen mit Migrationshintergrund. Drei davon sind diejenigen, die selbst einen Migrationshintergrund (in zwei Fällen mit Eltern aus Polen, in einem mit Eltern aus der Türkei) haben. Die Führungskräfte mit Migrationshintergrund zeigen auch ansonsten ähnliche Einstellungen wie die Führungskräfte ohne Migrationshintergrund, wenn diese sich unterschiedlicher Herausforderungen und Themenschwerpunkte für Personen mit und ohne Migrationshintergrund erster Generation bewusst sind. Gleichzeitig sind sie sich der Möglichkeit, aktiv auf die Diversity-Kultur einzuwirken, nicht bewusst, genauso wenig wie die meisten anderen Befragten.

\subsection{Welche Erfahrungen sind bei MINT-Führungskräften für die Einstellungen zu kultureller Vielfalt besonders relevant?}

Im Zuge der Interviews äußerten die Befragten verschiedene Erfahrungen, die ihre Sichtweise auf interkulturelle Interaktionen und kulturelle Vielfalt geprägt zu haben scheinen. Zunächst kann man einen Unterschied zwischen den Einstellungen derjenigen Führungskräfte beobachten, die viel mit internationalen Partner/innen, Kund/ innen oder Kolleg/innen zusammenarbeiten, und jenen, die dies nicht tun. Solche, die besonders viel international agieren, insbesondere die Führungskräfte aus dem IT-Bereich und aus den besonders großen Unternehmen, haben in vielen Fällen eine culture-blind-Einstellung, aber gleichzeitig konkrete Handlungsstrategien zum Umgang mit kultureller Vielfalt. Diese acht Befragten mit vielen internationalen Kontakten sind zwar sehr auf die Interaktion mit Ausländer/innen fokussiert, während Migrierte der zweiten Generation als weniger relevant betrachtet werden. Der Fokus liegt weniger darauf, interkulturelle Unterschiede zu verstehen, als vielmehr darauf, einzelne Fett-Näpfchen zu vermeiden. Dazu wird in drei Fällen auch eine agile Arbeitsweise und das Rahmenwerk Scrum erwähnt, das eine Gleichbehandlung aller Teammitglieder vorsehe. Agiles Arbeiten fasst eine extrem populäre Reihe methodischer Frameworks zusammen, die das Ziel verfolgen, effizientes und flexibles 
Projektmanagement im Kontext von VUCA zu ermöglichen (Schwaber und Sutherland 2020). Jedoch scheint kein Bewusstsein für Themen zum proaktiven Umgang mit kultureller Vielfalt innerhalb der Unternehmen vorhanden zu sein.

Führungskräfte, die in ihrer täglichen Arbeit nur wenig mit internationalen Kontakten oder anderen Personen mit Migrationshintergrund interagieren (obwohl das Unternehmen durchaus internationale Märkte bedienen kann), zeigen eine deutlich negativere Einstellung zu kultureller Vielfalt. Alle 12 Führungskräfte, die den Eindruck haben, dass sich kulturelle Subgruppen in ihren Unternehmen gebildet haben, haben in ihrem persönlichen Arbeitsalltag nur sehr wenig mit Personen mit Migrationshintergrund zu tun. Gleichzeitig sind diese Führungskräfte wesentlich weniger sicher, was interkulturelle Führung erfordert und was Personen mit und ohne Migrationshintergrund tun können, um erfolgreich zu interagieren. Man geht davon aus, dass Personen mit Migrationshintergrund in jedem Fall Deutsch lernen müssen.

\subsection{Welche Einstellungen und Perspektiven im sozialen Umfeld sind bei MINT- Führungskräften für die Einstellungen zu kultureller Vielfalt besonders relevant?}

Der Schluss liegt nahe, dass die eigenen negativen Einstellungen, bzw. die eigene Unsicherheit und mangelnde Fähigkeiten, mit übergeordneten Instanzen legitimiert werden: ,ja, also, ich sage einmal, in den Führungsebenen würden mit Sicherheit nicht hier im Hause akzeptieren, wenn auf einmal der Teppich nach Mekka ausgerichtet wird. Das ist einem deutschen Familienunternehmen, deutsch geführt, nicht akzeptabel von der Geschäftsführung, also: das würden die nie machen“. Bei vertiefter Betrachtung der Interviews zeigt sich, dass die meisten Befragten als Vertreter/ innen des jeweiligen Unternehmens auftreten. Die Führungskräfte argumentieren alle, bis auf zwei, aus der Perspektive des Unternehmens, wenn es um personelle Entscheidungen geht (z.B. „Wir haben da noch nicht so viel gemacht; also, jeder kann zu uns kommen“), oder beispielsweise um den Umgang mit Fehlern (z.B. „Bei uns wird das so gehandhabt ..."). Je häufiger Indizien für eine starke Identifikation auftreten, desto stärker scheinen die Führungskräfte sich selbst als typische Vertreter/innen des Unternehmens zu sehen, insbesondere im Hinblick auf den kulturellen Hintergrund. Abweichungen, vor allem kulturelle Abweichungen, scheinen kritischer gesehen zu werden, wenn die Identifikation hoch ist:

„Nach welchen Kriterien, neben der fachlichen Eignung, wählen Sie Ihre akademischen Mitarbeiter aus?“ „,̈hm ja, auf jeden Fall Sprache Deutsch, Sprachqualität (...) in Wort und Schrift, ganz wichtig. EDV heißt auch Kommunikation, und das sind nicht einfach irgendwelche Aufgaben, die im Hinterzimmer gemacht werden müssen, sondern man muss mit den Leuten sprechen und kommunizieren können. Und wir sind ein deutsches Familienunternehmen, von daher ja, ich sage einmal, wir sind lokal eigentlich sehr eingeschränkt. Wir haben zwar Niederlassungen in Frankreich und in Polen, aber auch dort wird an den Führungsstellen auf jeden Fall Deutsch gesprochen.“

Bei den vier Befragten, die nicht nur abstrakte Vorteile in kultureller Diversität sehen, sondern kulturelle Vielfalt als konkrete Stärke ihres eigenen Unternehmens 
auffassen, ist keine derartige Eigengruppen-Projektion zu beobachten. Das gilt auch für diejenigen Führungskräfte, die eine kulturell heterogene Belegschaft haben oder viel international interagieren. Darüber hinaus scheint die Haltung des Unternehmens eine Rolle zu spielen. Bei den Führungskräften, die ein positiveres und differenzierteres Bild von kultureller Vielfalt und interkultureller Kommunikation hatten, war in sechs Fällen auch von Unternehmensseite mehr unternommen worden, um die Diversität zu unterstützen oder gleichwertige Bedingungen zu schaffen.

\subsection{Welche Kompetenzen und welches Knowhow sind bei MINT- Führungskräften vorhanden und für die Einstellungen zu kultureller Vielfalt besonders relevant?}

Die Befragten zeigen insgesamt ein heterogenes Verständnis von Kompetenzen, die für interkulturelle Führung notwendig sind. Wie bereits erläutert wurde, ist bei den Führungskräften, die Vielfalt als Stärke des Unternehmens betrachten, die schon verschiedene Diversity-Management-Maßnahmen erlebt haben und in deren Unternehmen international interagiert wird, ein differenzierteres Bild vom Umgang mit kultureller Vielfalt vorhanden, das sich in konkreteren Antworten zeigt. Die Führungskräfte sind bis auf einen Befragten der Überzeugung, dass sie selbst gut interkulturell führen und ausreichend sensibel auf die Angestellten reagieren. Die Führungskräfte mit Migrationshintergrund unterscheiden sich dabei nicht in ihrem Antwortverhalten von Führungskräften ohne Migrationshintergrund. Obwohl sie sensibler sind als die meisten anderen Befragten, scheinen sie nicht unbedingt differenzierter über die Thematik nachzudenken. Dies könnte ein Hinweis darauf sein, dass Sensibilität zwar ein notwendiger Bestandteil eines kompetenten Umgangs mit Vielfalt ist, aber möglicherweise darüber hinaus auch Wissen und ,praktische Fähigkeiten“ erfordert. Dies deckt sich mit den Aussagen von elf der Befragten, dass Nachwuchs-Führungskräfte oft Probleme in diesem Bereich haben. Fünf weisen explizit darauf hin, dass sie dies als Defizit in der universitären Ausbildung empfinden:

Mehr Tools, mehr Ausbildung. Ich sagte ja eingangs auch, [interkulturelle] Führung ist an der Uni so gut wie gar nicht existent, zumindest ich habe das überhaupt nicht als Fachauswahl gehabt, nicht einmal als Wahlpflichtfach. Und ich kriege das auch bei anderen gespiegelt. Und wenn ich einmal so überlege, was ich in den letzten Jahren an Dingen gelernt habe, nehmen Sie einmal Friedemann Schulz von Thun, sein peer one-Modell, oder die verschiedenen Ebenen der Kommunikation und so weiter und so fort, all das sind eigentlich Basics, also wirklich Basics, die man normalerweise schon in die, ich sage einmal, in die fünfte bis zehnte Klasse packen müsste. Weil das einfach unheimlich wichtig ist im weiteren Fortkommen. Und Führung muss einfach an die Unis, und das muss eines der wichtigsten Fächer sein, denn man hat nachher mit Menschen zu tun, überall.

Diejenigen, die diesen Punkt im Verlauf der Interviews besonders betont haben, haben überwiegend selbst verschiedene Schulungen in "soft-skills“ in Eigenregie oder mit Unterstützung durch das Unternehmen absolviert. Bei den Führungskräften, bei denen das nicht der Fall ist, ist oft auch nur wenig Bewusstsein dafür vorhanden, 
dass man Angestellte und Führungskräfte auf interkulturelle Führung vorbereiten kann. Dass dies in anderen Fachbereichen der Standard sein könnte, wird in keinem der Interviews aufgegriffen, obwohl immer wieder darauf hingewiesen wird, dass man eigentlich zu wenig darüber wisse.

\section{Diskussion}

Die Ergebnisse zeigen, dass sich nur ein kleiner Teil der Befragten der Chancen und Risiken kultureller Vielfalt und der spezifischen Herausforderungen für Menschen mit Migrationshintergrund bewusst ist. Dabei scheinen für die Einstellungen gegenüber kultureller Vielfalt sowohl die eigenen Erfahrungen und die Sensibilität als auch die Position des Unternehmens und bisherige Maßnahmen relevant zu sein und miteinander zu interagieren. So zeigen sich für die Befragten unterschiedliche Muster, wenn sie viel oder wenig mit Personen mit Migrationshintergrund interagieren und wenn sie sich mehr oder weniger mit ihrem Unternehmen identifizieren. Außerdem zeigen diejenigen, deren Unternehmen Diversity-Maßnahmen etabliert haben, andere und differenziertere Einstellungen zu kultureller Vielfalt. Diese Ergebnisse knüpfen größtenteils an bestehende Ergebnisse zu Einstellungen zu kultureller Vielfalt an (van Dick und Stegmann 2016; Gutentag et al. 2018; Genkova und Schreiber 2019), was impliziert, dass sich Führungskräfte aus dem MINT-Bereich hinsichtlich Diversity-Einstellungen durchaus mit denen aus anderen Branchen vergleichen lassen, obwohl Stereotype von intelligenten, aber unempathischen Männern in MINTBerufen das Gegenteil suggerieren. Interindividuelle Unterschiede zwischen den Befragten scheinen jedoch auf den Arbeitskontext und systematische Defizite in Wissen und Handlungskompetenz im Umgang mit kultureller Vielfalt zurückzuführen $\mathrm{zu}$ sein und weniger auf die Zugehörigkeit zur MINT-Branche oder auf besonders starke Übereinstimmungen mit dem stereotyp MINT.

Diejenigen Führungskräfte, die wenig mit Personen mit Migrationshintergrund zusammenarbeiten, zeigen weniger konkrete Kompetenzen für den Umgang mit kultureller Vielfalt, sind weniger sensibel gegenüber Herausforderungen für Personen mit Migrationshintergrund und vertreten gleichzeitig eher eine culture-blindPerspektive. Mit dem Argument, dass alle Menschen gleich seien, begründen sie, warum Vielfalt an sich wichtig sei, aber weil sie alle gleichbehandeln, hätten sie damit kein Problem. Dieses Ergebnis deckt sich mit den Ergebnissen von Genkova und Schreiber (2019), dass viele Führungskräfte aus allen Branchen Herausforderungen im Arbeitsalltag ihrer Angestellten gar nicht wahrnehmen. Diejenigen, die diese negativeren Einstellungen in dieser Studie zeigen, sind ebenfalls alle der Meinung, für die Zugehörigkeit zur Organisation müssen Bewerber/innen Deutsch sprechen. Pehrson et al. (2009) zeigen anhand einer Querschnittstudie, dass der Zusammenhang zwischen nationaler Identität und Vorurteilen gegenüber Ausländer/innen stärker ist, wenn Nationalität an der Ethnie oder der Sprache festgemacht wird, verglichen dazu, wenn Nationalität über Staatsbürgerschaft definiert ist. Dass die Teilnehmenden aus Unternehmensperspektive sprechen und Vorbehalte mit betrieblichen Belangen verknüpfen, legt nahe, dass auch die Identifikation mit dem Unternehmen zu einer negativeren und undifferenzierten Einstellung gegenüber Personen mit Migrations- 
hintergrund führen kann, wenn die deutsche Sprache als relevanter Teil der Unternehmenskultur wahrgenommen wird. Zukünftige Studien sollten dies überprüfen.

Diejenigen, die Kontakt zu Personen mit Migrationshintergrund haben, sind nicht notwendigerweise sensibler gegenüber Personen mit Migrationshintergrund oder kompetenter im Umgang. Dieses Bewusstsein ist jedoch vorhanden, wenn bereits Maßnahmen für die Unterstützung von Diversity durchgeführt wurden. Es ist naheliegend, dass eine klare Positionierung von Unternehmen ,,pro Diversity“ bei einer hohen Identifikation mit dem Unternehmen dazu führt, dass positive und differenzierte Einstellungen eher übernommen würden, was den Ergebnissen von Ashikali und Groeneveld (2015) entspräche. Bei hoher Identifikation und fehlender oder negativer Positionierung des Unternehmens ist immer wieder von „denen“ die Rede, wenn es um Personen mit Migrationshintergrund geht. Personen mit Migrationshintergrund werden als organisations- oder Team-extern wahrgenommen. In Anlehnung an Mummendey und Wenzel (1999) lässt sich diese Einschätzung auf die Eigengruppenprojektionshypothese zurückführen, die besagt, dass Personen eine übergeordnete Gruppe, wie etwa ein Unternehmen, als homogener und ähnlicher zu sich selbst erleben, als es eigentlich ist. Diese Perspektive fanden auch Gutentag et al. (2018) bei der Untersuchung von Einstellungen zu kultureller Vielfalt und zeigen, dass einerseits Vorurteile und Fremdenfeindlichkeit Grund für culture-blindness sein können, aber auch das Gefühl, von der Komplexität des Themas überfordert zu sein. Generell scheinen die meisten der befragten MINT-Führungskräfte zumindest in der Vergangenheit festgestellt zu haben, dass das Thema kulturelle Vielfalt zwar präsent, aber sehr komplex ist und dass das vorhandene Wissen dazu nicht ausreicht. Es wird auch darauf hingewiesen, dass in MINT-Studiengängen kein Umgang mit kultureller Vielfalt gelehrt wird, obwohl nicht darauf eingegangen wird, dass andere Studiengänge dies standardmäßig machen. Die Befragten scheinen sich darin zu unterscheiden, wie sie mit dieser Unsicherheit umgehen. Dies passt zu der Tendenz einiger befragter MINT-Führungskräfte, die eigenen Ansichten mit der Haltung des Managements oder des Unternehmens zu legitimieren, was als Zeichen für Unsicherheit gewertet werden kann. Bei diesen Führungskräften scheint es einen eher resignativen Umgang mit der Herausforderung kultureller Vielfalt zu geben. Andere Führungskräfte (insbesondere aus größeren Unternehmen) lösen die Problematik, indem sie sich weiterbilden. Dies kann möglicherweise an einem besseren Zugang $\mathrm{zu}$ Weiterbildungsangeboten in großen Unternehmen liegen, aber möglicherweise auch daran, dass dort auf der Führungsebene eine größere Vielfalt an Fachbereichen in der Führungsebene anzutreffen ist und öfter eine klare Unternehmenslinie vorhanden ist.

Für alle Befragten zeigt sich, dass die Führungskräfte dem Thema kulturelle Vielfalt eher Zeit und Aufmerksamkeit geben, wenn es in der Unternehmensidentität subjektiv verankert ist. Für diesen Zusammenhang zwischen Unternehmenspositionierung, Weiterbildungsmöglichkeiten und Kompetenzerleben spricht ebenfalls, dass diejenigen Führungskräfte, die sich der Problematik und der Herausforderungen eher bewusst sind, auch verstärkt der Meinung sind, dass Führung allgemein und interkulturelle Führung insbesondere einer besonderen Ausbildung bzw. Weiterbildung bedürfen, die nur partiell durch Erfahrung substituiert werden kann. Es wird betont, dass Nachwuchs-Führungskräfte die Anforderungen interkultureller Führung 
oft nicht erfüllen und einen nicht unproblematischen Try-and-Error Prozess durchlaufen, bis sie störungsfrei arbeiten können. Dies entspricht auch den branchenübergreifenden Beobachtungen von Barmeyer et al. (2019). Ein zentrales Ergebnis dieser Studie ist daher, dass sowohl von Unternehmens- wie von Universitätsseite eine stärkere Berücksichtigung von kultureller Vielfalt erforderlich ist, um den Anforderungen der kulturell heterogenen Gesellschaft entsprechen zu können.

Die Analyse der Interviews zeigt außerdem, dass agiles Arbeiten und DiversitySensibilität nicht notwendigerweise miteinander einhergehen. McCallaghan et al. (2020) demonstrieren anhand einer Querschnittsstudie, dass servant leadership (unterstützende Führung), ein Kernkonzept agiler Frameworks, positiv mit den Diversity-Einstellungen der Angestellten zusammenhängt. Sie weisen jedoch auch darauf hin, dass sie Diversity-Einstellungen lediglich als instrumentelle Komponente operationalisieren (also ob die Befragten der Meinung sind, Vielfalt stelle einen wirtschaftlichen Vorteil dar) und die Diversity-Sensibilität nicht erfassen. Wie auch Ashikali und Groeneveld (2015) zeigen sie, dass ein mitarbeitenden-orientierter Führungsstil es der Führungskraft ermöglicht, ihre Einstellungen weiterzugeben, was Vor- und Nachteile haben kann. Einerseits ist Gleichberechtigung sowohl in agilen Frameworks als auch im transformationalen Führungsstil fest verankert (Pusenius 2019). Andererseits gehen beide Konzepte nicht auf andere Facetten des Diversity-Managements ein. Die IT-ler/innen, die ihren Führungsstil als agil beschreiben, heben zwar Gleichheit hervor, haben aber kein vertieftes Wissen oder Bewusstsein für die Chancen und Risiken kultureller Vielfalt. Obwohl für diese Einstellung auch die übrigen Mechanismen, die in dieser Arbeit umrissen wurden, wirken könnten, scheint ein Zusammenhang naheliegend, und weitere Studien sollten eine Verbindung von agilen Methoden und Diversity-Einstellungen adressieren, besonders in Anbetracht der zunehmenden Verbreitung agiler Arbeitsweisen.

\subsection{Limitationen}

Die vorliegende Studie ist mit Limitationen behaftet. Frauen und Männer zeigten keine Unterschiede im Antwortverhalten, eine separate Betrachtung möglicher Zusammenhänge von Einstellungen und Erfahrungen wurde jedoch nicht vorgenommen. Obwohl die Perspektiven der Führungskräfte mit Migrationshintergrund gesondert betrachtet wurden, ist es wahrscheinlich, dass diese Studie auch hinsichtlich dieses Aspekts nicht auf andere Personen übertragen werden kann. Zukünftige Studien sollten diese Problematik beheben und konkret Frauen und Personen diversen Geschlechts sowie Personen mit Migrationshintergrund adressieren, auch um mögliche Effekte durch Intersektionalität zu berücksichtigen. Da kein geeignetes Vergleichsmaterial vorliegt, können keine Aussagen darüber getroffen werden, inwiefern die Teilnehmenden ein typisches oder generalisierbares Bild der MINT-Branche liefern. Auch über tatsächliche Fähigkeiten und Eigenschaften lässt sich kein Schluss ziehen, da dies z.B. zusätzlich eine Befragung von Mitarbeitenden und Kunden erfordert hätte. Die Ergebnisse legen jedoch nahe, dass die Defizite, die die befragten MINTFührungskräfte hinsichtlich Diversity-Bewusstsein und Kompetenzen aufzuweisen scheinen, überwiegend durch den Arbeitskontext und mangelnde Ausbildung entstehen als durch eine spezifische Prädisposition von Menschen in MINT-Berufen. 
Ob dieses Ergebnis auch für Mitarbeitende in der MINT-Branche zutrifft, sollte in weiteren Studien überprüft werden. Darüber hinaus bietet es sich für weitere Studien dieser Art an, nicht nur Perspektiven und Meinungen abzufragen, sondern diese mittels situativer Fragen konkretisieren zu lassen. Während einige Befragte ihre Erörterungen mit vielen Beispielen untermauerten, waren andere sehr zurückhaltend, gerade bei negativen Erfahrungen.

\subsection{Fazit}

Die vorliegende Studie konnte trotz ihrer Limitationen relevante Zusammenhänge für die weitere Forschung im Bereich Diversity und Diversity-Management explorieren. Besonders hervorzuheben ist, dass Führungskräfte keineswegs als reine Vermittler zwischen einer übergeordneten Diversity-Strategie und den Angestellten stehen, wie Studien wie die von Ashikali und Groeneveld (2015) implizit annehmen. Für die Praxis bedeuten die Ergebnisse dieser Studie, dass Diversity-Management im Bereich MINT für die Unterstützung von Führungskräften sehr relevant ist, dass Führungskräfte mit dem notwendigen Wissen und Verständnis ausgestattet werden müssen, um effizient und angemessen handeln zu können und dies auch an ihre Angestellten weitergeben zu können. Die vorliegenden Ergebnisse geben Hinweise darauf, dass agiles Arbeiten kein Diversity-Bewusstsein schafft. Auch bei Universitätsabsolvent/ innen kann nicht davon ausgegangen werden, dass in diesem Bereich Erfahrungen bestehen. Die globalisierte und digitalisierte Arbeitswelt macht es für das DiversityManagement unerlässlich, diese Einstellungen und Einflüsse auf Führungskräfte zu berücksichtigen, will man das Wie und Warum kultureller Vielfalt in MINT-Berufen verstehen.

Funding Open Access funding enabled and organized by Projekt DEAL.

Open Access Dieser Artikel wird unter der Creative Commons Namensnennung 4.0 International Lizenz veröffentlicht, welche die Nutzung, Vervielfältigung, Bearbeitung, Verbreitung und Wiedergabe in jeglichem Medium und Format erlaubt, sofern Sie den/die ursprünglichen Autor(en) und die Quelle ordnungsgemäß nennen, einen Link zur Creative Commons Lizenz beifügen und angeben, ob Änderungen vorgenommen wurden.

Die in diesem Artikel enthaltenen Bilder und sonstiges Drittmaterial unterliegen ebenfalls der genannten Creative Commons Lizenz, sofern sich aus der Abbildungslegende nichts anderes ergibt. Sofern das betreffende Material nicht unter der genannten Creative Commons Lizenz steht und die betreffende Handlung nicht nach gesetzlichen Vorschriften erlaubt ist, ist für die oben aufgeführten Weiterverwendungen des Materials die Einwilligung des jeweiligen Rechteinhabers einzuholen.

Weitere Details zur Lizenz entnehmen Sie bitte der Lizenzinformation auf http://creativecommons.org/ licenses/by/4.0/deed.de.

\section{Literatur}

Anger, C., Koppel, O., \& Plünnecke, A. (2018). MINT-Frühjahrsreport 2018: MINT - Offenheit, Chancen, Innovationen. https://www.arbeitgeber.de/www/arbeitgeber.nsf/res/MINT-Fruehjahrsreport_ 2018.pdf/. Zugegriffen: 19.10.2021. 
Ashikali, T., \& Groeneveld, S. (2015). Diversity management in public organizations and its effect on employees' affective commitment. Review of Public Personnel Administration, 35(2), 146-168. https:// doi.org/10.1177/0734371X13511088.

Barmeyer, C., Bausch, M., \& Moncayo, D. (2019). Cross-cultural management research: topics, paradigms, and methods-A journal-based longitudinal analysis between 2001 and 2018. International Journal of Cross Cultural Management, 19(2), 218-244. https://doi.org/10.1177/1470595819859603.

Bengtsson, M. (2016). How to plan and perform a qualitative study using content analysis. NursingPlus Open, 2, 8-14. https://doi.org/10.1016/j.npls.2016.01.001.

Canagarajah, S. (2018). Materializing 'competence': Perspectives from international STEM scholars. The Modern Language Journal, 102(2), 268-291.

Cox, T. (1991). The multicultural organization. Academy of Management Perspectives, 5(2), 34-47. https:// doi.org/10.5465/AME.1991.4274675.

van Dick, R., \& Stegmann, S. (2016). Diversity, Social Identity und Diversitätsüberzeugungen. In P. Genkova \& T. Ringeisen (Hrsg.), Handbuch Diversity Kompetenz (S. 3-15). Wiesbaden: Springer. https:// doi.org/10.1007/978-3-658-08594-0_6.

Genkova, P. (2019). Diversity und Diversity Management. In P. Genkova (Hrsg.), Interkulturelle Wirtschaftspsychologie (S. 351-364). Berlin: Springer. https://doi.org/10.1007/978-3-662-58447-7_16.

Genkova, P., \& Schreiber, H. (2019). Diversity potentials in organizations. In B. Covarubias-Venengas, C. Debray \& J. Vakkayil (Hrsg.), IACCM research conference: intercultural competencies for a disruptive VUCA world: exploring creativity, innovation, resilience \& resistance in intercultural research, training \& management. Symposium im Rahmen der Tagung von IACCM. Paris: IACCM.

Gutentag, T., Horenczyk, G., \& Tatar, M. (2018). Teachers' approaches toward cultural diversity predict diversity-related burnout and self-efficacy. Journal of Teacher Education, 69(4), 408-419. https://doi. org/10.1177/0022487117714244.

Hilmer, J. (1969). Grundzüge einer pädagogischen Theorie der Bewegungsspiele. Ein Beispiel zur Didaktik der Leibeserziehung. Hannover: Schroedel.

Kemper, T. (2010). Migrationshintergrund - eine Frage der Definition! Die Deutsche Schule, 102(4), Artikel 4, 315-326. https://www.pedocs.de/frontdoor.php?source_opus=5151. Zugegriffen: 19.10.2021.

van Knippenberg, D., \& Haslam, S. A. (2003). Realizing the diversity dividend: exploring thesubtle interplay between identity, ideology, and reality. In S. A. Haslam, D. van Knippenberg, M. J. Platow \& N. Ellemers (Hrsg.), Social identity at work: developing theory for organizational practice (S. 61-77). Hove: Psychology Press.

van Knippenberg, D., \& Schie, E.C.M. (2000). Foci and correlates of organizational identification. Journal of Occupational and Organizational Psychology, 73(2), 137-147. https://doi.org/10.1348/ 096317900166949.

van Knippenberg, D., Haslam, S. A., \& Platow, M.J. (2004). Unity through diversity: value-in-diversity beliefs, work group diversity, and group identification. Group Dynamics: Theory, Research, and Practice, 11(3), 207-222. https://doi.org/10.1037/1089-2699.11.3.207.

Koller, K., \& Rudolph, C. (Hrsg.). (2017). Gender Mainstreaming und Diversity in der (akademischen) MINT-Weiterbildung: Konzepte, Prozesse und Befunde. Regensburg: OTHmind - BMBF-Verbundprojekt.

Kundu, S. C., \& Mor, A. (2017). Workforce diversity and organizational performance: a study of IT industry in India. Employee Relations, 39(2), 160-183. https://doi.org/10.1108/ER-06-2015-0114.

Mayring, P. (2015). Qualitative Inhaltsanalyse: Grundlagen und Techniken (12. Aufl.). Weinheim: Beltz.

McCallaghan, S., Jackson, L. T.B., \& Heyns, M. M. (2020). Servant leadership, diversity climate, and organisational citizenship behaviour at a selection of South African companies. Journal of Psychology in Africa, 30(5), 379-383. https://doi.org/10.1080/14330237.2020.1821310.

Mummendey, A., \& Wenzel, M. (1999). Social discrimination and tolerance in intergroup relations: Reactions to intergroup difference. Personality and Social Psychology Review, 3(2), Art. 3, 577-595.

Pehrson, S., Vignoles, V.L., \& Brown, R. (2009). National identification and anti-immigrant prejudice: individual and contextual effects of national definitions. Social Psychology Quarterly, 72(1), 24-38. https://doi.org/10.1177/019027250907200104.

Pusenius, K. (2019). Agile mindset in the workplace: moving towards organizational agility. Master's Thesis. : Turku University of Applied Sciences.

Schein, E.H. (2010). Organizational culture and leadership (4. Aufl.). Hillsdale: Erlbaum.

Schwaber, K., \& Sutherland, J. (2020). The Scrum guide: the definitive guide to Scrum: the rules of the game. https://www.scrumguides.org/docs/scrumguide/v2020/2020-Scrum-Guide-US.pdf\# zoom=100. Zugegriffen 19.10.2021. 
Stegmann, S. (2011). Engaging with diversity of social units. Dissertation. Frankfurt am Main: Johann Wolfgang Goethe-Universität.

Tajfel, H., \& Turner, J. (1979). An integrative theory of intergroup conflict. In W. G. Austin \& S. Worchel (Hrsg.), The social psychology of intergroup relations (S. 33-47). Belmont: Brooks/Cole.

Thomas, A. (2003). Interkulturelle Kompetenz. Grundlagen, Probleme und Konzepte. Erwägen - Wissen - Ethik, 14(1), 137-228.

Wildermuth, C., \& Gray, S. (2005). Diversity training. Alexandria: ASTD Press.

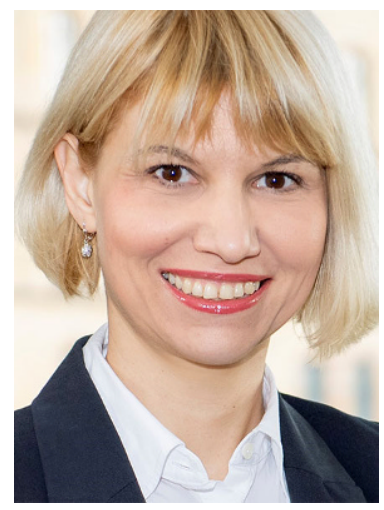

Prof. Dr. Petia Genkova Prof. Dr. phil. habil., ist Professorin für Wirtschaftspsychologie an der Hochschule Osnabrück und leitet mehrere Forschungsprojekte zu den Themen Interkulturelle Kompetenz, Erleben, Gesundheit und Zufriedenheit von Personen mit Migrationshintergrund sowie Diversity. Darüber hinaus ist sie Trägerin des Höffmann-Wissenschaftspreises für Interkulturelle Kompetenz für ihr Lebenswerk.

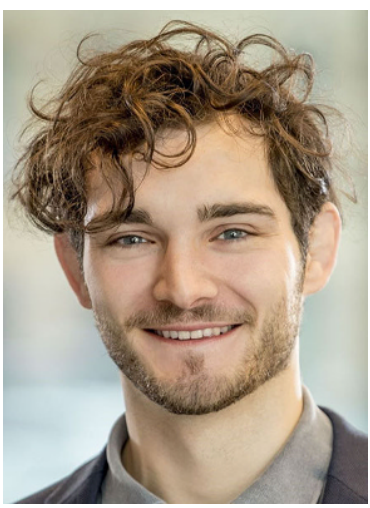

Henrik Schreiber ist wissenschaftlicher Mitarbeiter an der Hochschule Osnabrück. Seine Forschungsbereiche umfassen interkulturelle Kompetenz sowie den individuellen und organisationalen Umgang mit Diversität, insbesondere Diversity Beliefs. 